\title{
Store Elements LabView-based Energy-flow Models Developmnet and Implementation in HVAC systems
}

\author{
Szász Csaba \\ Department of Electrical Machines and Drives \\ Technical University of Cluj \\ Csaba.Szasz@emd.utcluj.ro
}

\begin{abstract}
It is well known that store elements such as liquid tanks, water reservoirs, or gas containers, are basic elements in heating, ventilation and air conditioning (HVAC) systems development and implementation. In a wide range of applications they serve as efficient energy storage elements by capturing heat energy amount in hot water form. This paper presents a LabView graphical software-based energy-flow model development for store elements in HVAC systems. In the first research steps the mathematical background describing the energy-flow processes in the considered store element (water tank) it is presented and discussed. Then an original software model has been designed and implemented to simulate the ongoing energy-flow processes. The result of the above mentioned research efforts is a powerful and versatile software toolkit (virtual instrument) well suitable to modeling and simulate complex energy-flow processes in HVAC systems embedding various types of store elements. Beside the elaborated mathematical model concrete software simulation examples and measurement data are also provided in the paper. Not at least, the proposed original model offers a feasible solution for future developments and research in HVAC systems software modeling and simulation purposes.
\end{abstract}

Keywords - store element, energy-flow, HVAC system, LabView model, virtual instrument

\section{INTRODUCTION}

As it is well known, a wide range of computer software is available on the market to guide and help researches in complex HVAC systems design and implementation purposes. These tools with a varying degree of complexity and performance have been developed for different scopes and applications, and for different user or customer categories [1]. Some existing HVAC systems modeling and simulation toolkits ensures the user that the respectively tool is easy to use and fulfills in all the daily needs and requirements of designer's. On the other side, there are complex software toolkits providing an accurate prediction of system or components performance. In other words, this generous offer embeds a large scale of software products starting with toolkits used by engineers to automate common tasks, continuing with computer aided drafting (CAD) programs to modeling and simulate operating conditions (i.e. to predict annual energy use and utilization expenses), and up to specialized analysis tools $[1,2]$. These last types of software resources are used as research instruments including technically accurate simulation modules specially developed for academic study and researches in HVAC systems development and implementation, usually focused on details and performance criteria.

Obviously, the above discussed computer software resources may be classified in several main groups or categories. According to P. Jacobs and H. Henderson, it can be distinguished the following major categories [2]:

- Practitioner Design Tools (attempt to automate common or repetitive tasks such as drawing, sizing, analyzing, costing, and are used by professionals to design HVAC systems);

- Whole Building Energy Analysis Tools (aimed at simulate building energy use and determinate operating conditions across the year, calculating annual operating expenses);

- Energy and Environmental Screening Tools (focused to evaluate cost-effectiveness and environmental impact of energy- and HVAC technologies);

- Specialised Analysis Tools (embedding the best available methods and techniques to simulate specific aspects of building or energy system performance; that are generally not in widespread use by professionals).

However, it is no doubt among specialists and researchers involved that the established HVAC system models and simulation toolkits play a key role in the entire building automation systems development and implementation process. Of course, as it has been mentioned above, there are on the market a wide range of currently available products, ranging from spread-sheet tools to special-purpose tools, or tools that integrate multiple aspects of HVAC systems modeling and simulation [1, 3]. There is no enough space to enumerate or discuss all these toolkits (and it is not the scope of this paper), but it is outlined that the current fourth generation tools tend to be fully integrated with respect to different intelligent buildings performance aspects, such as automate monitoring and supervising, application quality control, intelligent user interfaces, fault-diagnosis, etc. Unfortunately, even at this lastgeneration level there are important discussions among 
researchers involved in HVAC systems development regarding the adopted models and simulation strategies [3]. It seems that some basic HVAC element or component models are not enough well established and developed yet, or fully clarified. There is the room where this paper proposes to add contributions and solutions by developing and implementing novel LabView software-based models suitable to improve the HVAC systems modelling and simulation processes.

\section{STORE ELEMENTS ENERGY-FLOW MODEL DEVELOPMENT}

It is well known that store elements (various types of water tanks, gas containers, or other liquid tanks) are widely used elements in HVAC systems development and implementation. Usually they serve as efficient energy store elements by capturing heat energy amount. For the research purposes appointed in this paper it is available one 7501 capacity specially developed hot water buffer tank manufactured by the Hajdú company (Fig. 1).

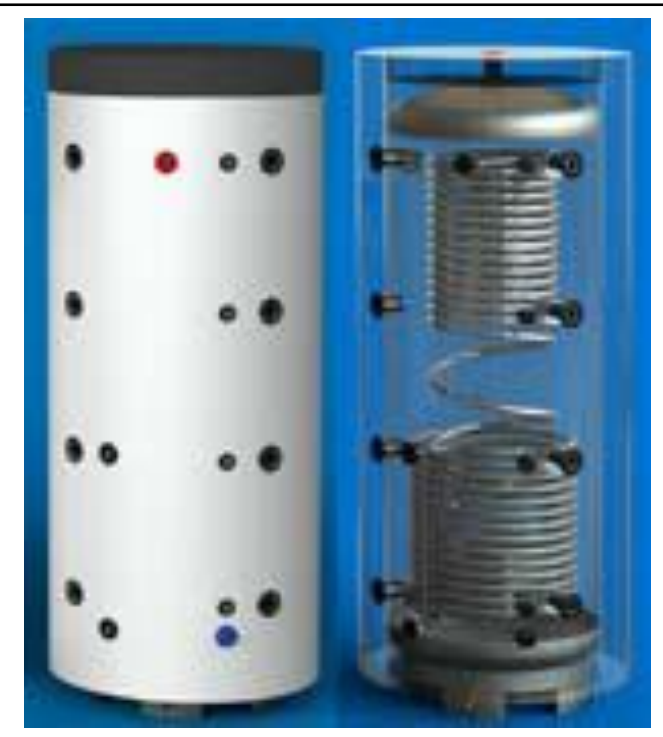

Fig. 1. The water buffer tank manufactured by the Hajdú company (Debrecen University, Technical Faculty, Department of Electrical Engineering and Mechatronics)

This basic HVAC element it is used in a heating and airconditioning system to store in a form of a hot water the heat energy amount captured by a forced air-duct heat pump system installed in a building. In order to develop the energyflow model of this store element, it is considered the simplified block diagram shown below in "Fig. 2".

In the entire design process it will be taken into account that the stored hot water will be used both for heating and cooling

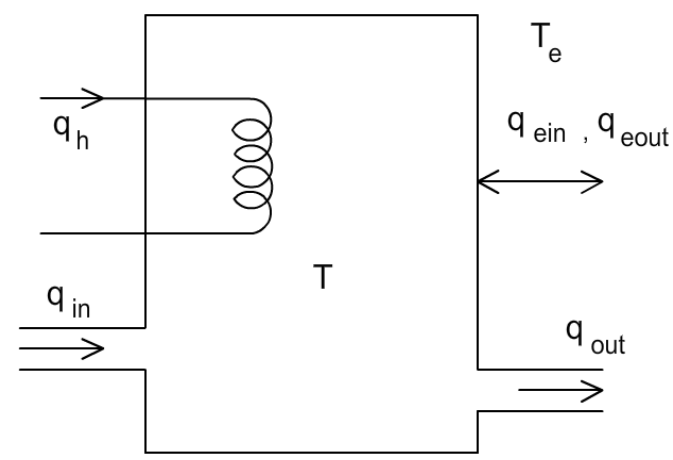

Fig. 2. Energy-flow model of a store element (hot water tank) purposes. Toward, it is assumed that the water temperature is the same everywhere inside the tank (ideal mixing process). In order to calculate the instantaneous temperature $(T)$ of the stored water, it is necessary to solve the following energy balance equation [3]

$$
m \cdot c_{w} \cdot \frac{d T}{d t}=\rho \cdot V \cdot c_{w} \cdot \frac{d T}{d t}=q_{n}
$$

where $q_{n}[\mathrm{~kW}]$ represents the net heat energy transfer to the tank water, $c_{w}[\mathrm{~kJ} / \mathrm{kgK}]$ is the specific heat capacity of the water, $V\left[\mathrm{~m}^{3}\right]$ is the tank volume, and $m[\mathrm{~kg}]$ the mass of the stored water. Additionally, it is given the $4,1813[\mathrm{~kJ} / \mathrm{kgK}]$ specific heat capacity of the water at $25^{\circ} \mathrm{C}$ temperature. The net $q_{n}$ heat energy transfer can be calculated by considering all the energy-flow processes between the tank and its environment. Therefore, it can be written the following relationship [3]:

$q_{n}=q_{h}+q_{\text {out }}+q_{\text {in }}+q_{\text {ein }}+q_{\text {eout }}+q_{\text {pin }}+q_{\text {pout }}$,

where the given magnitudes are with the following interpretation:

- $\quad q_{h}$ heat added by the heater element or burner;

- $q_{\text {out }}$ is the heat transfer to/from the use side plant connections;

- $q_{\text {in }}$ is the heat transfer to/from the source side plant connections;

- $q_{\text {ein }}$ heat transfer to/from the ambient environment $(0$, when the heater is turned off);

- $q_{\text {eout }}$ heat transfer to/from the ambient environment $(0$, when the heater is turned on);

- $q_{\text {pin }}$ heat added due to on-cycle parasitic loads ( 0 , when the heater is turned off);

- $q_{\text {pout }}$ heat added due to off-cycle parasitic loads (0, when the heater is turned on);

Due to simplify calculations in this model the $q_{\text {pin }}$ and $q_{\text {pout }}$ parasitic heat amounts will be neglected by considering ideal on/off switching processes. Therefore, the equation (2) simplifies to the following relationship:

$$
q_{n}=q_{h}+q_{\text {out }}+q_{\text {in }}+q_{\text {ein }}+q_{\text {eout }}
$$

The heat quantities changed by the water thank to/from its near environment can be expressed with the equations:

$$
q_{\text {ein }}=k_{\text {ein }} \cdot\left(T_{e}-T\right) ; q_{\text {eout }}=k_{\text {eout }} \cdot\left(T_{e}-T\right) \text {, }
$$

where $T_{e}$ means the environment temperature where the tank is placed, $k_{\text {ein }}$ és $k_{\text {eout }}$ are the loss coefficients of the given water tank in on/off operation mode. The $q_{\text {out }}$ and $q_{\text {in }}$ heat energyflows are calculated with the mathematical expressions [3]: 


$$
\begin{aligned}
& q_{\text {out }}=k_{\text {out }} \cdot m_{\text {out }} \cdot c_{w} \cdot\left(T_{\text {out }}-T\right) ; q_{\text {in }}=k_{\text {in }} \cdot m_{\text {in }} \cdot c_{w} \cdot \\
& \left(T_{\text {in }}-T\right) \text {, }
\end{aligned}
$$

$k_{\text {out }}$ and $k_{\text {in }}$ being efficiency coefficients in the given relations. At the same time $T_{\text {out }}$ means the temperature on the user side plant, supposing that the buffer tanks even may be placed in other rooms (for example: cellar or basement) like the user heated ones. The $T_{i n}$ variable represents the temperature of the recycled water into the tank. Carrying out the adequate substitutions in the equation (3), results:

$q_{n}=q_{h}+k_{\text {out }} \cdot m_{\text {out }} \cdot c_{w} \cdot\left(T_{\text {out }}-T\right)+k_{\text {in }} \cdot m_{\text {in }} \cdot c_{w}$
$\left(T_{\text {in }}-T\right)+k_{\text {ein }} \cdot\left(T_{e}-T\right)+k_{\text {eout }} \cdot\left(T_{e}-T\right)$.

After performing the simple mathematical operations results that:

$$
\begin{gathered}
\frac{d T}{d t}=\frac{1}{m \cdot c_{w}} \cdot\left(q_{h}+k_{\text {out }} \cdot m_{\text {out }} \cdot c_{w} \cdot T_{\text {out }}+k_{\text {in }} \cdot m_{\text {in }} \cdot c_{w}\right. \\
\left.\cdot T_{\text {in }}+k_{\text {ein }} \cdot T_{e}+k_{\text {eout }} \cdot T_{e}\right)+ \\
+\frac{-1}{m \cdot c_{w}} \cdot\left(k_{\text {out }} \cdot m_{\text {out }}^{\cdot} \cdot c_{w}+k_{\text {in }} \cdot m_{\text {in }} \cdot c_{w}+k_{\text {ein }}+k_{\text {eout }}\right) \\
\cdot T .
\end{gathered}
$$

The above relationship is nothing else than a

$$
\frac{d T}{d t}=a+b \cdot T
$$

type differential equation, where

$$
\begin{gathered}
a=\frac{1}{m \cdot c_{w}} \cdot\left(q_{h}+k_{\text {out }} \cdot m_{\text {out }} \cdot c_{w} \cdot T_{\text {out }}+k_{\text {in }} \cdot m_{\text {in }} \cdot c_{w}\right. \\
\left.\cdot T_{\text {in }}+k_{\text {ein }} \cdot T_{e}+k_{\text {eout }} \cdot T_{e}\right) \\
b=\frac{-1}{m \cdot c_{w}} \cdot\left(k_{\text {out }} \cdot m_{\text {out }} \cdot c_{w}+k_{\text {in }} \cdot m_{\text {in }} \cdot c_{w}+k_{\text {ein }}+k_{\text {eout }}\right) \\
\cdot T .
\end{gathered}
$$

The solution of the equation 8 is [3]:

$$
T(t)=\left(\frac{a}{b}+T_{v k}\right) \cdot e^{b t}-\frac{a}{b}
$$

$T_{w i}$ meaning the water initial temperature at the moment $t=0$. If the $b$ coefficient is zero, then the solution of the differential equation is:

$$
T(t)=a \cdot t+T_{w i}
$$

If it is necessary to calculate the amount of time in which the water reaches its $T_{w f}$ final temperature, in a same way from the relationship 8 results the following value:

$$
t=\frac{1}{b} \cdot \ln \left[\frac{\left(\frac{a}{b}+T_{w f}\right)}{\left(\frac{a}{b}+T_{w i}\right)}\right] .
$$

In the case of $b$ being zero

$$
t=\frac{\left(T_{w f}-T_{w i}\right)}{a} .
$$

The above deduced mathematical relations are sufficient to describe the entire heating/cooling processes and energy-flow phenomena in store elements like water tanks or other liquid reservoirs.

\section{STORE ELEMENTS ENERGY-FLOW PROCESSES LABVIEW- BASED MODELLING AND SIMULATION}

By using as background the mathematical model developed in the previous paragraph, original LabView graphical software-based virtual instruments (VIs) have been designed and implemented in order to reproduce and simulate the energy-flow processes in store elements. As an example, in "Fig. 3" it is presented the energy-flow model of a 7501 capacity water buffer tank. Hot water flows into the tank via the adequate pipe system (Boolean_1) carrying the heat energy generated by the air-water heat pump mounted in the building's ceiling. The hot water is circulated continuously by using the Boolean_2 pump and the opened Boolean_3 valve. It means that the heater (or burner) of the tank it is switched into the on state, the stored water is heated now. The red colour of the pipes indicates a hot water flow, the green colour of the pump and valve the operation mode, respectively the open-state of these devices. If the heating process is stopped, the pipes turns instantaneously into a gray colour, and the pump and valve off states will be marked with red colours. The red pipe mounted on the right-down side of the water tank indicate that the hot water it is used for indoors heating purposes and the tank it is connected to fan coils placed in different rooms of the buildings. The left-down side placed blue colored pipe expresses that the hot water is circulated inside a closed pipe system, into the store element returning cool water, after the heat amount has been transmitted to the indoors environment via the used fan coil system. The input heat amount can be easily controlled by using the left side evidenced slide-switch controller.

For the energy-flow process simulation it is necessary to provide only the $T_{w i}$ initial temperature of the water amount stored in the tank and to open the slide-switch heat controller. In this case the stored water instantaneous temperature will be indicated both by a numerical display and graphical thermometer placed on the Front Panel of the VI. Additionally, the temperature variation is plotted via a virtual oscilloscope in order to ensure a convenient monitoring facility of this magnitude evolution in a given time domain. Toward, a numerical indicator displays the instantaneous heat amount in watts stored in the water tank respectively a $L E D$ diode indicates any change of the slide-switch position which controls the input heat amount. 
In fig. 4 it is given the simulation Front Panel window the burner (or heater) yet is in the turn-off state.

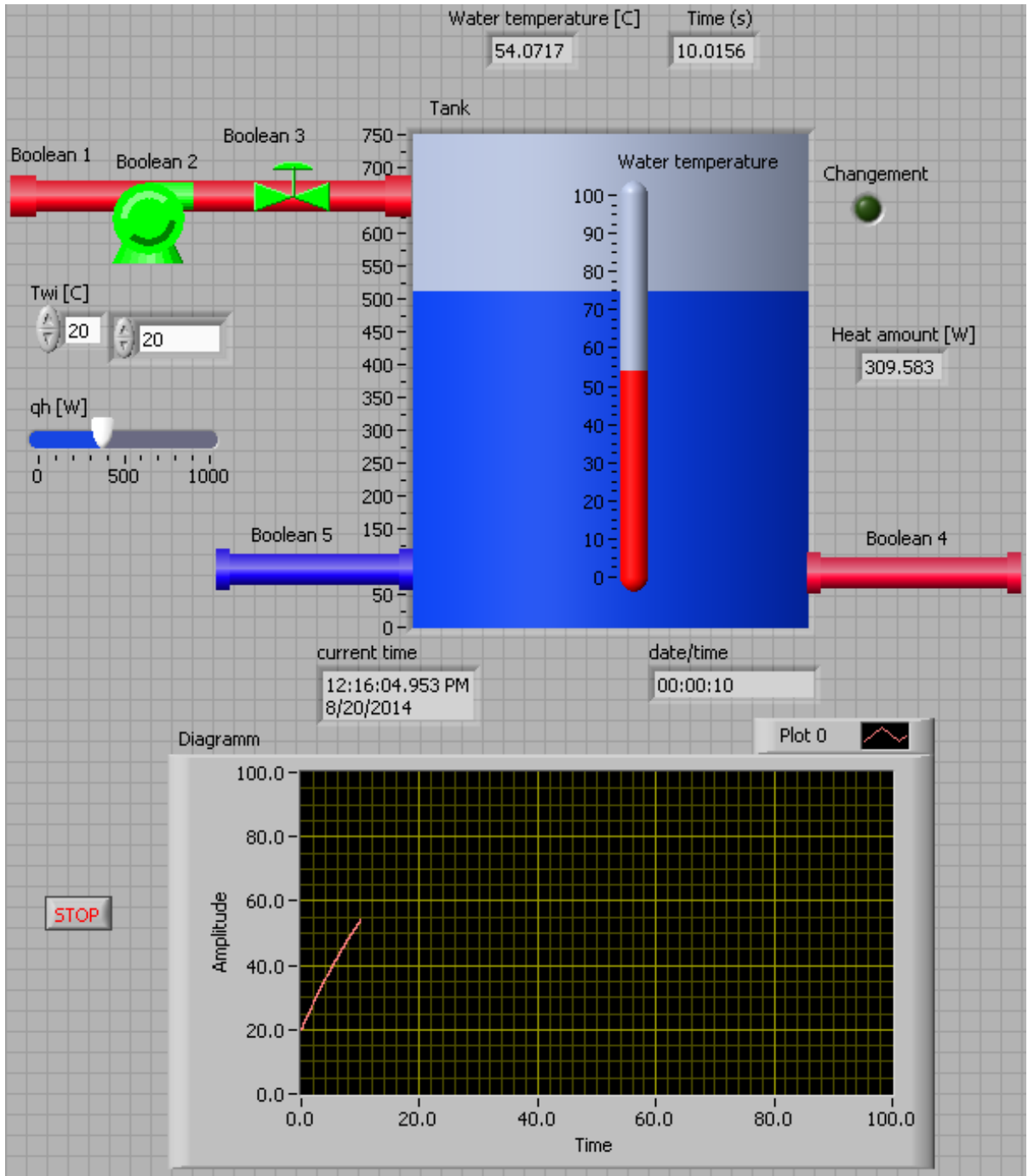

Figure 3. The store element energy-flow model (LabView-software, the VI Front Panel)

corresponding to the initial state of the hot water tank, when

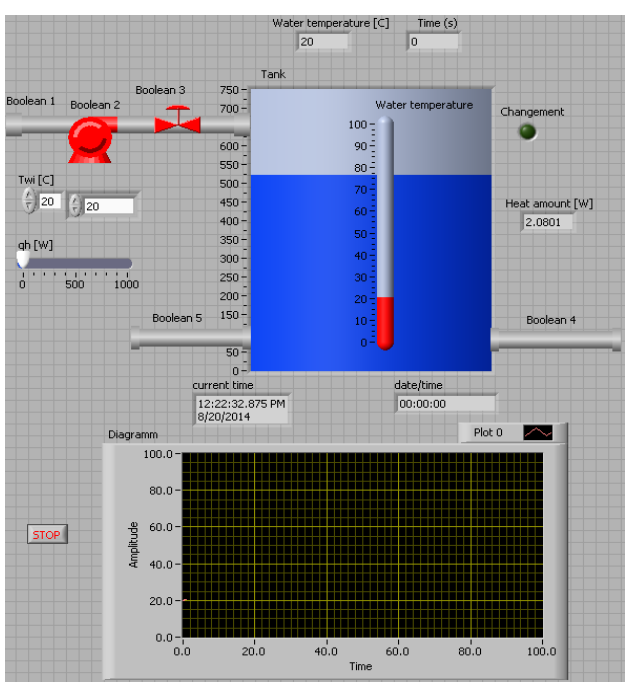

It is not without importance to mention here, that the developed energy-flow modelling and simulation software implements the catalogue parameters given by the Hajdu company. According to this, the model takes into account that the insulation of the tank is not ideally suited, therefore are considered its specific heat loss coefficients. Hence, when the outside environment temperature is lower than the stored water temperature automatically a heat transfer process will take place to the outside environment. Otherwise, when the stored water temperature is lower, the heat transfer process direction is reversed now. Other coefficients like specific heat capacities, flow-rates, mass flow, or other constants can be settled arbitrary (or after de user specific needs) in the LabView graphical program.

Figure 4. Initial state of the store element (hot water tank) 
In Fig. 5 it is given a piece from the LabView Block Diagram of the store element energy-flow modeling and simulation program. There it can be distinguished the mathematical equation used for implementation, VI units for events capturing, integration, display and monitoring, state machines implementation, or other embedded elements.

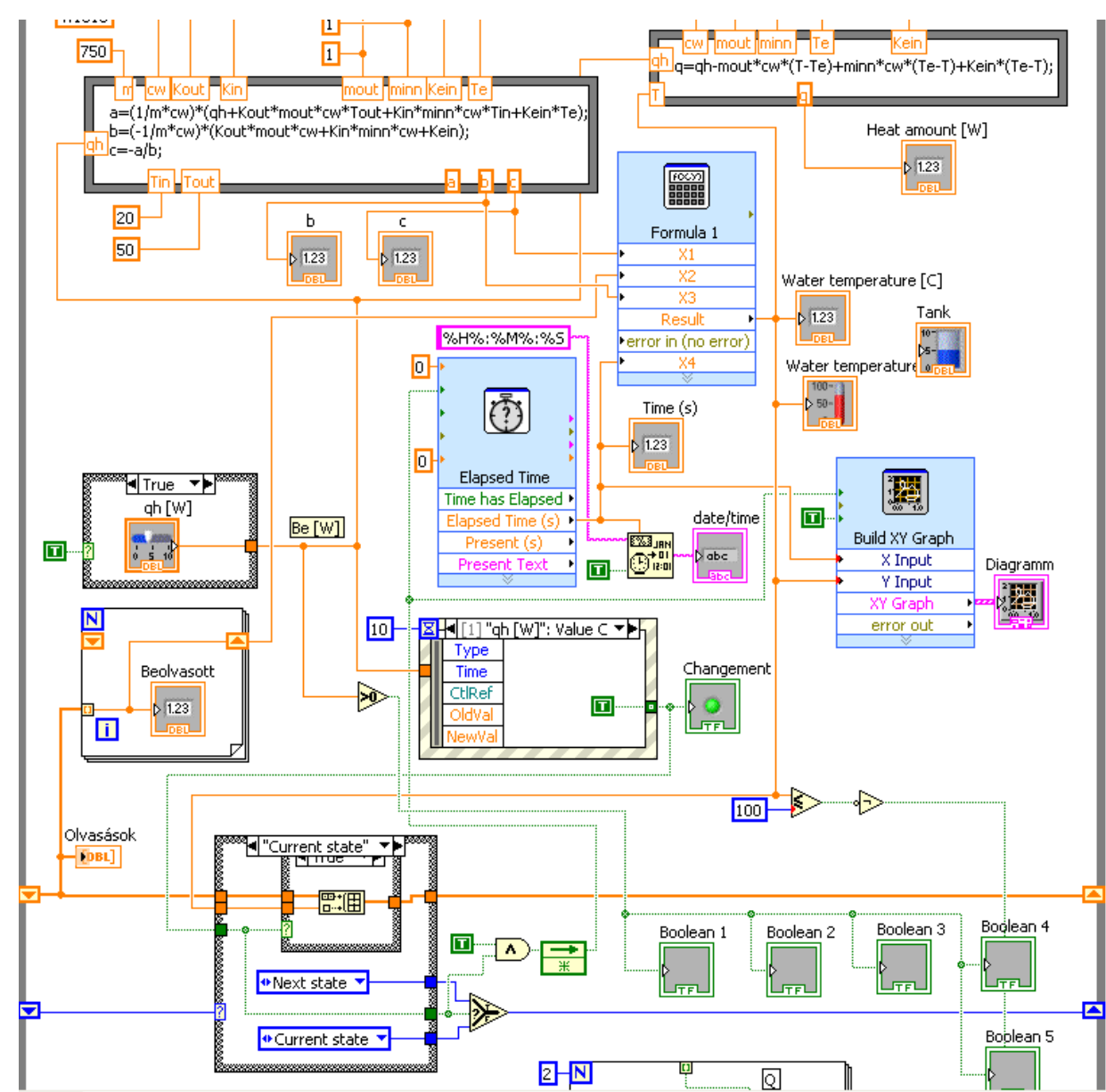

Figure 5. The store element energy-flow model LabView-based implementation (Block Diagram)

However, the developed virtual instrument allows sufficient flexibility and freedom for the users to model and simulate various energy-flow processes in a wide range of water tanks, gas containers, or other liquid tanks. Obviously, in this paper only a first version of the designed and implemented software has been presented. Taking into account the immense complexity of the addressed topic, this modelling and simulation software supports a continuously improvement and development process in order to achieve a more accurate imitation of the entire energy-flow processes in a wide range of store elements used in complex HVAC systems implementation.

\section{CONCLUSIONS}

The paper presents a LabView software-based energy-flow model development for store elements in HVAC systems. Beside the establishment of an adequate mathematical model indispensable for such a purpose, the software implementation steps of store elements energy-flow processes is unfolded and presented. The result is versatile and powerful software programs (VIs) suitable to simulate complex energy-flow processes and serving as novel tools for HVAC systems energy balance evaluation. Even in this stage of the research the elaborated software models are under a continuous development and improvement process, the proposed original model offers a feasible solution for future developments and research in HVAC systems modeling and simulation purposes.

\section{ACKNOWLEDGMENT}

This research was supported by the European Union and the State of Hungary, co-financed by the European Social Found in the framework of TÁMOP 4.2.4. A/2-11-1-20120001 'National Excellence Program'.

\section{REFERENCES}

[1] DOE, "Building Energy Software Tools Directory", U.S. Department of energy, available at: http://www.eere.energy.gov/ [accessed: August, 2009]. 
Recent Innovations in Mechatronics (RIiM) Vol. 2. (2015). No. 1-2. DOI: $10.17667 /$ riim.2015.1-2/18.

[2] J. Jacobs, H. Henderson, "State-of-the-art review whole building, building envelope, and HVAC component and system simulation and design tolls" US Department of Commerce, Port Royal Road, 5285, Springfield 22161, VA, 2002.

[3] EnergyPlus ${ }^{\mathrm{TM}}$-EnergyPlus Engineering Reference, "The reference to energy plus calculations" US Department of Energy, 2013. 